\title{
LincRNAs MONC and MIR100HG act as oncogenes in acute megakaryoblastic leukemia
}

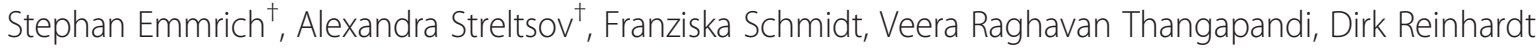 \\ and Jan-Henning Klusmann ${ }^{*}$
}

\begin{abstract}
Background: Long non-coding RNAs (IncRNAs) are recognized as pivotal players during developmental ontogenesis and pathogenesis of cancer. The intronic microRNA (miRNA) clusters miR-99a 125b-2 and miR-100 125b-1 promote progression of acute megakaryoblastic leukemia (AMKL), an aggressive form of hematologic cancers. The function of the IncRNA hostgenes MIR99AHG (alias MONC) and MIR100HG within this ncRNA ensemble remained elusive.

Results: Here we report that IncRNAs MONC and MIR100HG are highly expressed in AMKL blasts. The transcripts were mainly localized in the nucleus and their expression correlated with the corresponding miRNA clusters. Knockdown of MONC or MIR100HG impeded leukemic growth of AMKL cell lines and primary patient samples. The development of a lentiviral IncRNA vector to ectopically express IncRNAs without perturbing their secondary structure due to improper termination of the viral transcript, allowed us to study the function of MONC independent of the miRNAs in cord blood hematopoietic stem and progenitor cells (HSPCS). We could show that MONC interfered with hematopoietic lineage decisions and enhanced the proliferation of immature erythroid progenitor cells.
\end{abstract}

Conclusions: Our study reveals an unprecedented function of IncRNAs MONC and MIRTOOHG as regulators of hematopoiesis and oncogenes in the development of myeloid leukemia.

\section{Background}

It has become apparent that the vast majority of the eukaryotic genome underlies prevalent transcription [1]. Both DNA strands are pervasively transcribed, giving rise to numerous different classes of non-coding RNAs (ncRNAs), including long intergenic RNAs (lincRNAs), antisense RNAs and enhancer RNAs (eRNAs) [2]. This abundant mixture of long ( $>200 \mathrm{nt})$ and short $(<200 \mathrm{nt})$ non-coding RNAs was misapprehended in the past as transcriptional noise or junk. However, accumulating evidence suggested that transcription factors and other global regulators are prevalent targets of ncRNAs [3]. Thereby, ncRNAs induce changes in histone marks and gene expression in cis and in trans. For example, XIST is crucial for random inactivation of the X chromosome [4]. Beyond that, Xist RNA acts as a suppressor of hematologic cancer [5]. Deletion of Xist results in the development of a highly aggressive myeloproliferative neoplasm and

\footnotetext{
* Correspondence: Klusmann.Jan-Henning@mh-hannover.de

${ }^{\dagger}$ Equal contributors

Pediatric Hematology and Oncology, Hannover Medical School, Carl-Neuberg-Straße 1, 30625 Hannover, Germany
}

myelodysplastic syndrome. In contrast, HOTAIR regulates expression of the $H O X D$ gene family as well as other genes throughout the genome via re-targeting of Polycomb repressive complex 2 (PRC2) [6,7]. Enforced expression of HOTAIR in epithelial cancer cells leads to altered histone H3 lysine 27 methylation, gene expression, and increased cancer invasiveness and metastasis. Similarly, HOTTIP affects expression of the HOXA gene family [8]. Recently, E2F1 transcription factor has been shown to activate lncRNA ERIC, which restricts E2F-induced apoptosis during cell cycle progression [9].

Acute myeloid leukemia (AML) is an aggressive form of hematologic cancers with a 5-year overall survival between 30 and $40 \%$ in adults [10]. While AML is generally less common in children, inherited molecular lesions can cause a genetic background, which predisposes to malignant transformation and AML. Particularly children with Down syndrome (DS), i.e. trisomy 21 , have a 400 -fold increased risk [11] to develop acute megakaryoblastic leukemia (AMKL). Patients with DS-AMKL have an excellent prognosis with 5-year overall survival rates of about $80 \%$, while non-DS-AMKL patients have poor survival rates of only 
$14 \%$ to $34 \%$ despite high intensity chemotherapy $[12,13]$. The molecular mechanisms underlying this AML subtype remain incompletely understood. We recently reported the characterization of an oncogenic microRNA (miRNA) on chromosome 21 (hsa21), miR-125b-2, which is highly expressed in DS-AMKL and non-DS-AMKL. miR-125b-2 increased proliferation and self-renewal of human and mouse megakaryocytic progenitors (MPs) and megakaryocytic/erythroid progenitors (MEPs) [14]. This small RNA is located in a phylogenetically conserved ncRNA ensemble, consisting of two other miRNAs (miR-99a and let-7c) and the lncRNA hostgene MIR99AHG, which we termed megakaryocytic oncogenic non-coding RNA (MONC) (Figure 1A). A homolog of the miR-99a $\sim 125 b-2$ polycistron on hsa21 can be found in identical configuration in the intron of the lincRNA MIR100HG on hsa11 (miR-100 $125 b-1)$. We could previously demonstrate that miR-100 $125 b-1$ and miR-99a $\sim 125 b-2$ protect megakaryoblasts

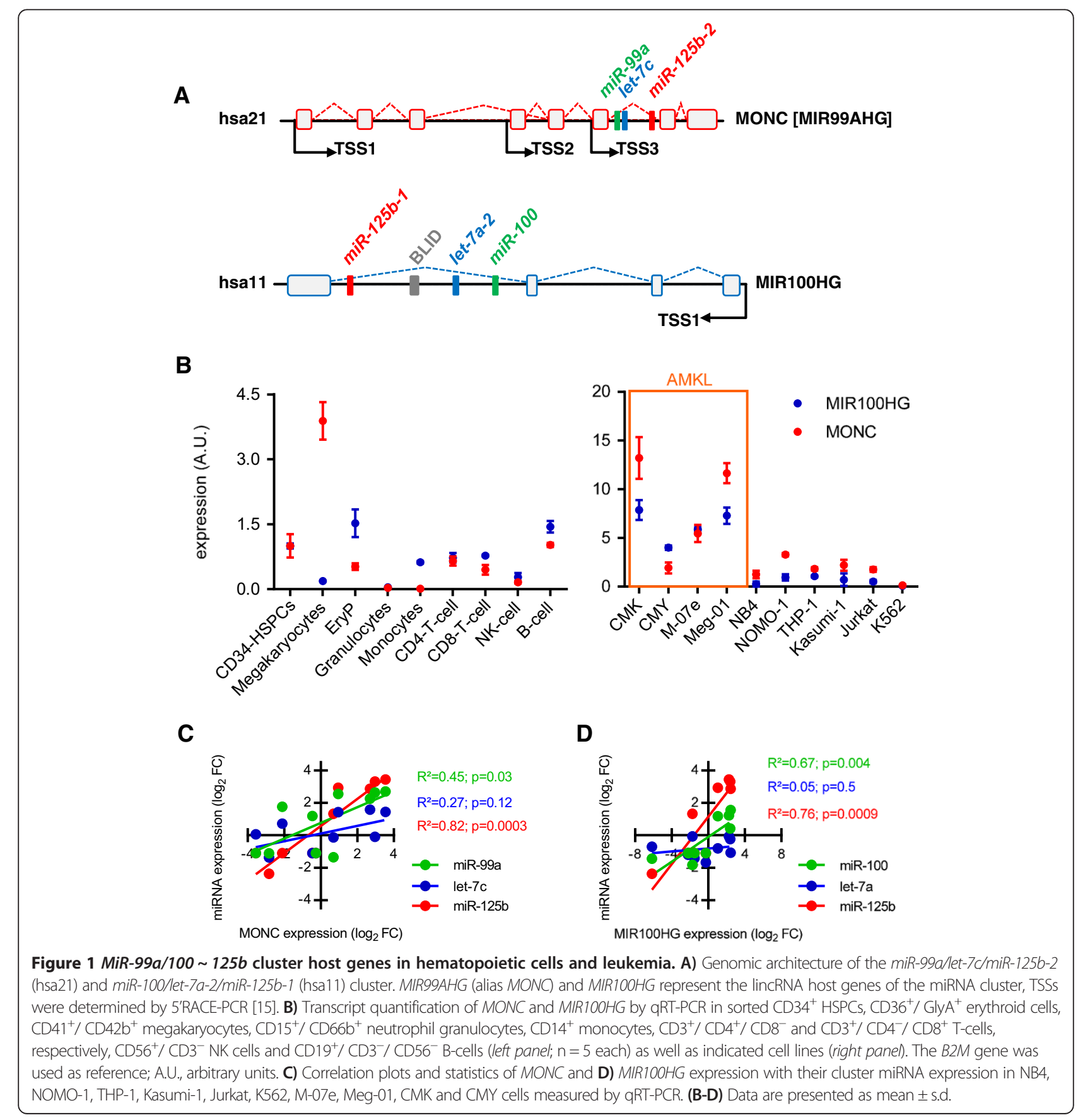


and leukemic cells from TGF $\beta 1$-mediated proliferation arrest and apoptosis [15]. However, the role of the lncRNA hostgenes in this ncRNA ensemble remained elusive.

In the present study, we characterized the function of MONC and MIR100HG and demonstrate an unprecedented role of lncRNAs MONC and MIR100HG during hematopoiesis and the pathogenesis of AMKL.

\section{Results \\ MiR-99a/100 125b cluster lincRNAs are overexpressed in AMKL}

The $m i R-99 a / 100 \sim 125 b$ clusters on hsa11 and hsa21 are central regulators of stem cell homeostasis and leukemogenesis and are hosted in introns of MIR100HG and $M O N C$, respectively. We mapped the transcriptional start sites (TSS) of both clusters by 5'RACE-PCR and demonstrated that the miRNAs are transcribed as one polycistronic transcript together with their host genes [15]. qRT-PCR expression profiling of spliced MONC and MIR100HG trancripts throughout hematopoietic lineages demonstrated higher expression of MONC in megakaryocytes, HSPCs and B-cells and higher expression of MIR100HG in erythroid cells, HSPCs and B-cells as compared to the other blood lineages (Figure 1B). Furthermore, MONC and MIR100HG are higher expressed in AMKL cell lines compared to various other leukemic cell lines (Figure 1B). Regression analysis confirmed positive correlation of MONC and MIR100HG with their respective miRNA polycistrons (Figure 1C,D). However, both mature let-7 isoforms did not show a strong positive correlation with their lincRNA host genes, suggesting active LIN28- and/or miR-107-mediated suppression of let-7 in MONC- and MIR100HG-high expressing cells [16,17].

Thus, expression patterns of spliced MONC and MIR100HG transcripts implicate an independent, yet unknown function in hematopoietic regulation and transformation.

\section{Knockdown of MIR100HG impairs cell proliferation and viability}

Therefore, we investigated the consequences of MIR100HG knockdown in the AMKL cell line Meg-01 with a high endogenous expression (Figure 1B). To achieve sufficient knockdown of endogenous MIR100HG, we designed two different shRNAs and verified a knockdown efficiency of $65 \%$ for sh-MIR100HG \#1 and 80\% for sh-MIR100HG \#2 by qRT-PCR (Additional file 1: Figure S1A).

Proliferation of Meg-01 cells was impaired upon MIR100HG knockdown (Figure 2A). In competition assays, where shMIR100HG-transduced Cerulean-positive $\left(\mathrm{Cer}^{+}\right)$Meg-01 cells were mixed with non-silencing control shRNAtransduced mCherry-positive $\left(\mathrm{mCh}^{+}\right) \mathrm{Meg}-01$ cells, both shRNAs against MIR100HG conferred a strong growth disadvantage (Figure 2B). In contrast, proliferation of
K562 cells with low to absent endogenous MIR100HG expression was mainly unaffected by sh-MIR100HGtransduction (Additional file 1: Figure S1B-C). The colonyforming capacity of Meg-01 cells was decreased upon MIR100HG-knockdown (Figure 2C). This effect was even aggravated in replating experiments for sh-MIR100HG \#2, the construct with the stronger knockdown efficacy (Figure 2D). In BrdU cell cycle analyses of Meg-01 cells, we observed an increase in the apoptotic subG1 fraction accompanied by a decrease of cycling cells in $S$ phase upon MIR100HG knockdown (Figure 2E). Accordingly, we monitored a significant increase of Annexin ${ }^{+}$apoptotic cells (Figure 2 F). Interestingly, MIR100HG knockdown changed the surface marker expression on the leukemic megakaryoblasts (Figure 2G). While the percentage of $\mathrm{CD}^{+} 6^{+}$cells increased from $11 \%$ in controls to $32 \%$, the percentage of $\mathrm{CD} 41^{+}$cells was $\sim 1.8$-fold reduced.

Taken together, knockdown of MIR100HG impaired cell viability and replating-efficiency of AMKL cells, while changing lineage surface marker expression.

Knockdown of MONC reduces cell proliferation and viability MONC is encoded on hsa21 and highly upregulated in both DS-AMKL (trisomy 21) and non-DS-AMKL cell lines (Figure 1B). Therefore we sought to evaluate the consequences of MONC knockdown in CMK and Meg-01 cell lines, representing those two entities. As a control, we used K562 cells with low to absent MONC expression (Figure 1B). We designed a total of 8 different shRNAs covering different sites of MONC. Only one shRNA had sufficient knockdown efficacy (Additional file 2: Figure S2A).

Cell proliferation was impaired by $M O N C$-knockdown in AMKL cells, yet was unaffected in K562 cells (Figure 3A, Additional file 2: Figure S2B). In growth competition assays we noticed a strong decline of $\mathrm{Cer}^{+}$sh-MONC-transduced AMKL cells (Figure 3B). Similarly, monitoring of cell growth by automated microscopy in K562, CMK and M-07 cell lines showed a reduction of sh-MONCtransduced AMKL cells, whereas their number was insignificantly changed in K562 cells (Additional file 2: Figure S2C). Accordingly, the colony-forming capacity of sh-MONC-transduced Meg-01 and CMK cells -but not K562 cells- was reduced (Figure 3C, Additional file 2: Figure S2D). Also replating experiments showed a decrease in the cumulative CFU number for both AMKL cell lines (Figure 3D). Cell cycle analysis demonstrated insignificant changes upon MONC knockdown (Figure 3E). sh-MONC favored apoptosis in Meg-01 but not in CMK cells as measured by Annexin V staining (Figure 3F). Quantification of megakaryocytic-erythroid surface markers (CD41 and CD36) revealed a reduction of $\mathrm{CD}^{+} 6^{+}$Meg-01 cells upon MONC knockdown (Figure 3G,H), while this effect was not observed in CMK cells. 


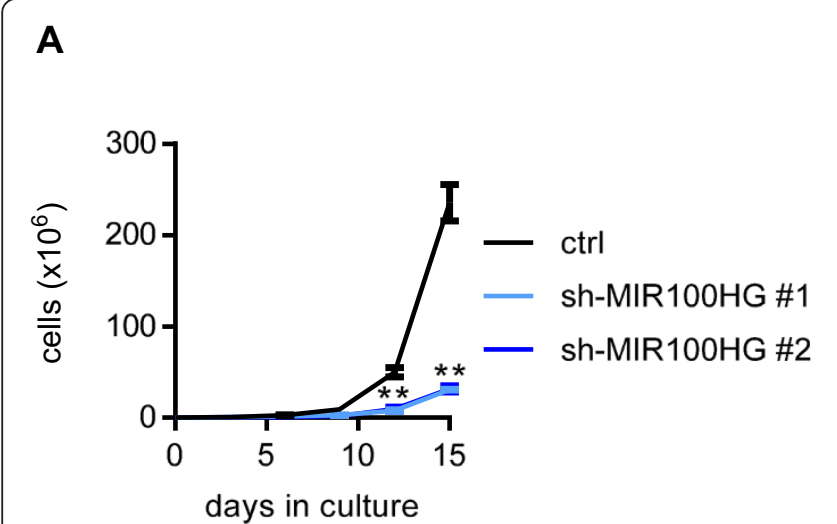

B
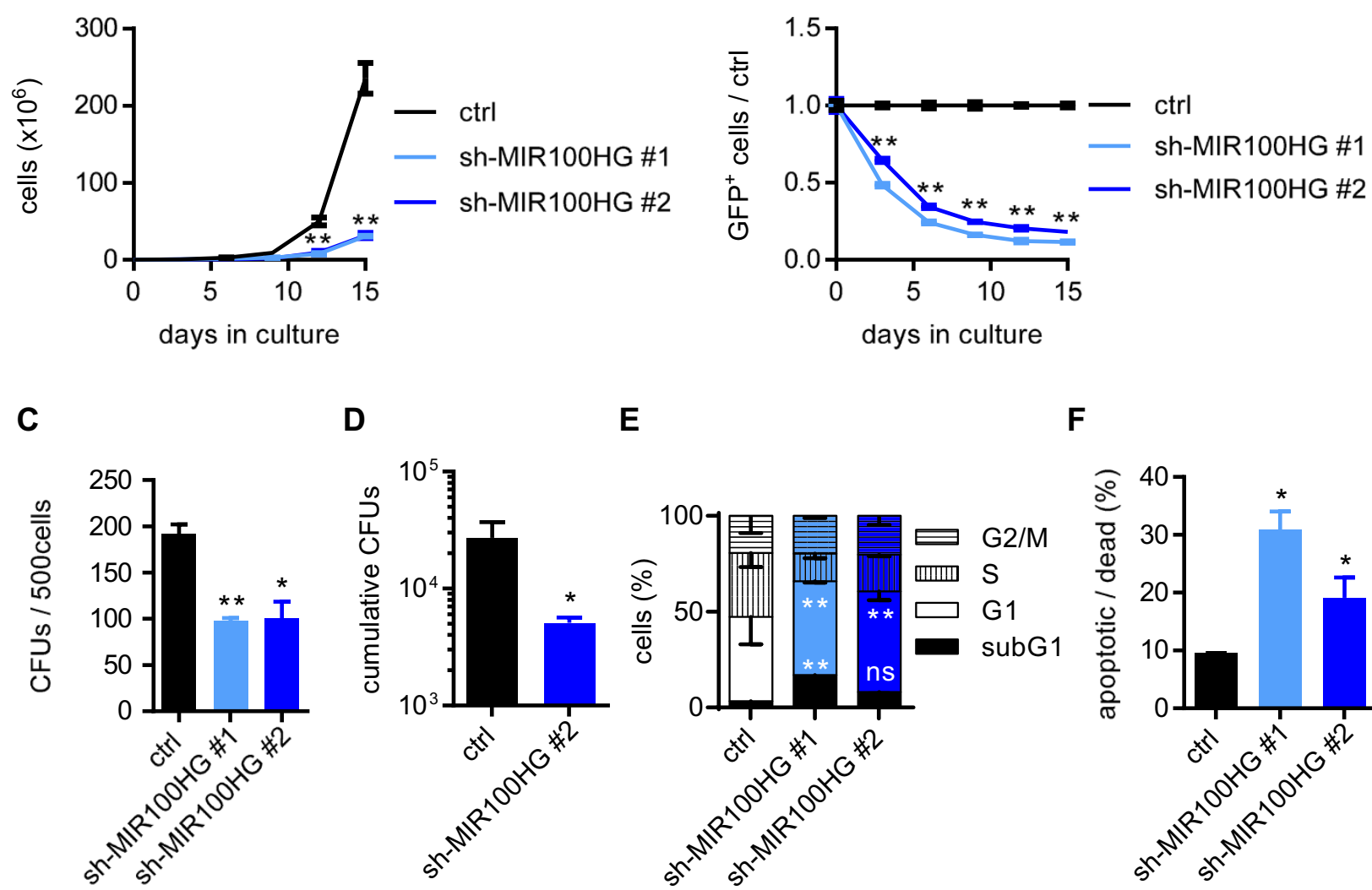

E

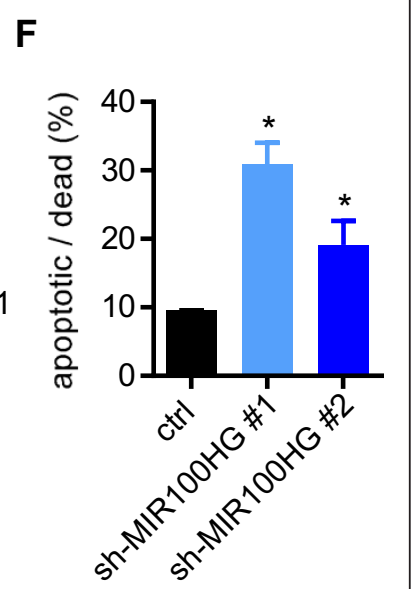

G
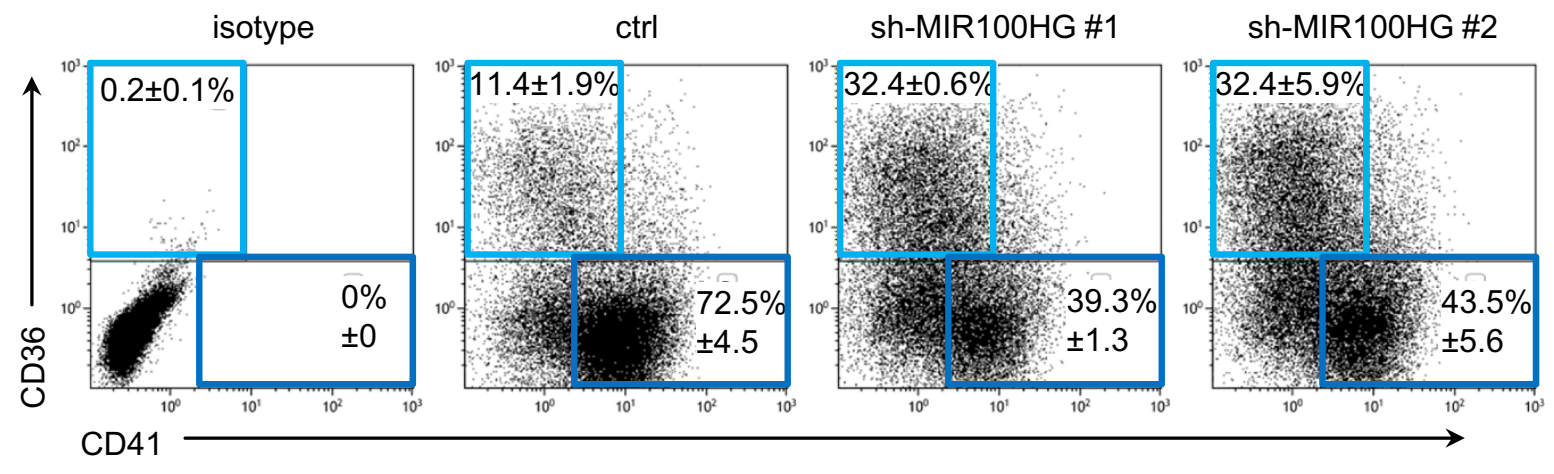

Figure 2 Knockdown of MIR100HG confers growth disadvantage to AMKL cells. A) Number of shRNA- or ctrl-transduced Meg-01 cells $(n=2)$. B) Fraction of Cerulean ${ }^{+}$shRNA-transduced cells at indicated time points of culture is shown in relation to the ctrl construct $(n=2$;

Two-way ANOVA was performed to compare the mean of each construct at each time point to ctrl). C) Number of colonies from methylcellulose-based CFU assays of shRNA-transduced Meg-01 cells $(n=2)$. D) Cumulative number of CFUs after one round of replating of sh-MIR100HG \#2 in Meg-01 cells

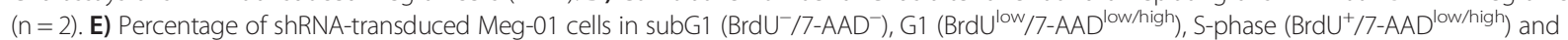
G2/M fraction (BrdU low/7-AAD high) $(n=2)$. Asterisks are indicated for subG1 and $S$ phases. F) Percentage of apoptotic/dead $\left(\right.$ Annexin $\left.V^{+}\right)$ shRNA-transduced Meg-01 cells after 5 days of culture $(n=2)$. G) Representative density plots of viable, Cerulean ${ }^{+}$Meg-01 cells for indicated surface markers as measured by flow cytometry after 5 days of culture $(n=4)$. (A-G) Data are presented as mean \pm s.d. ${ }^{*} P<0.05 ;{ }^{* *} P<0.01$.

Experiments in primary AML cells are challenging. However, they are pertinent to extrapolate observation made in cell lines to the situation in vivo. Strikingly, when DS transient leukemia (DS-TL) blasts were transduced with sh-MONC, colony-forming capacity was diminished (Figure 3I), implicating a role of hsa21-encoded MONC in the development and maintenance of trisomy 21associated leukemia. 


\section{B}
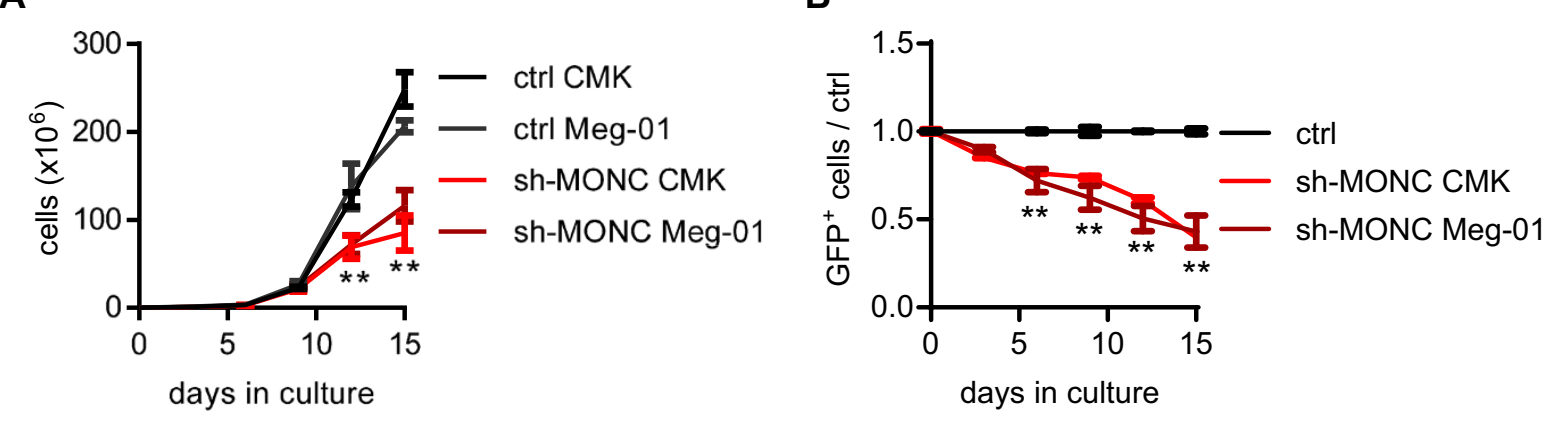

C

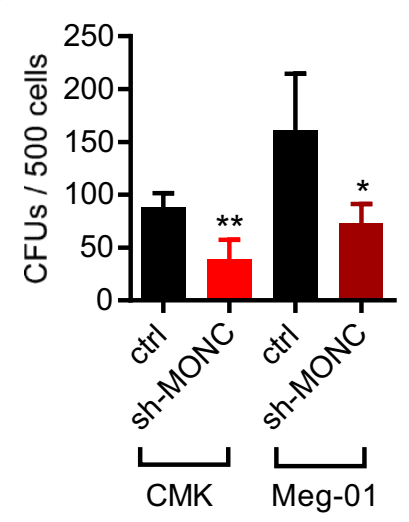

$\mathbf{F}$

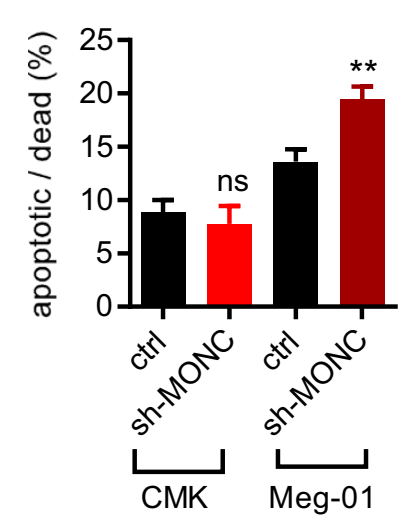

I

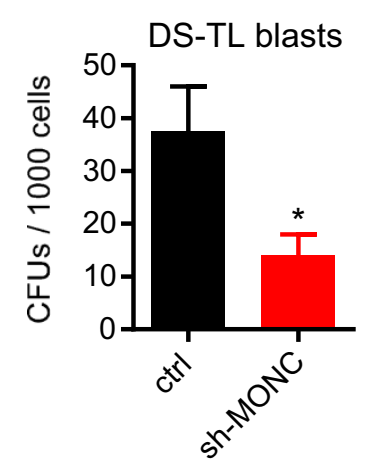

D

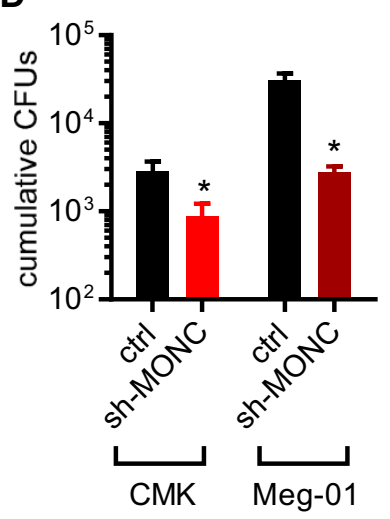

E

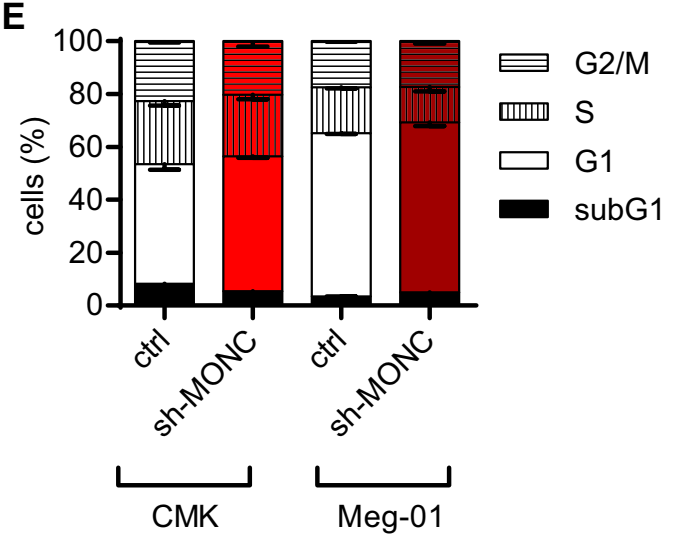

G
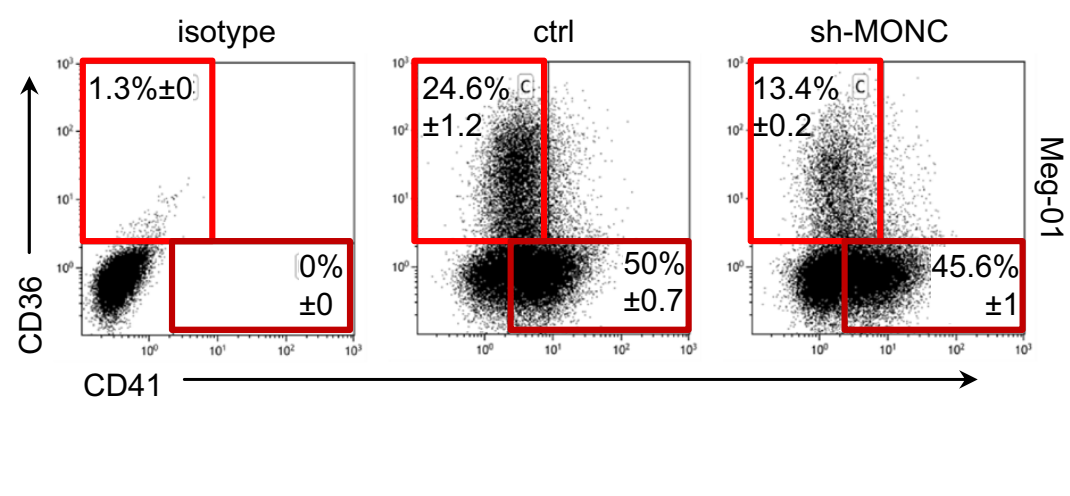

H

isotype

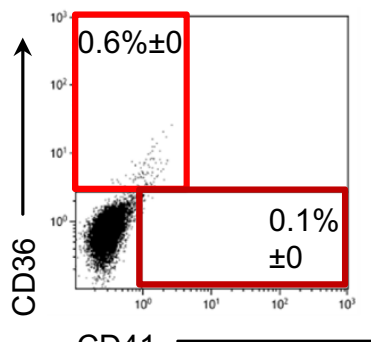

ctrl

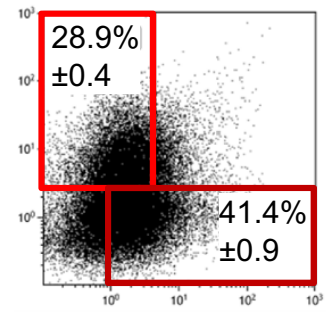

sh-MONC

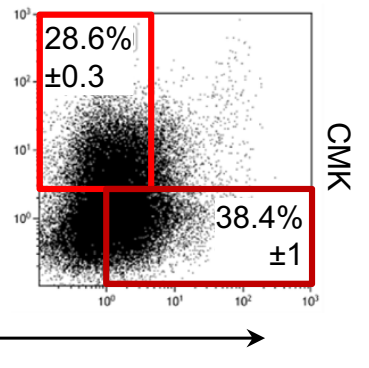




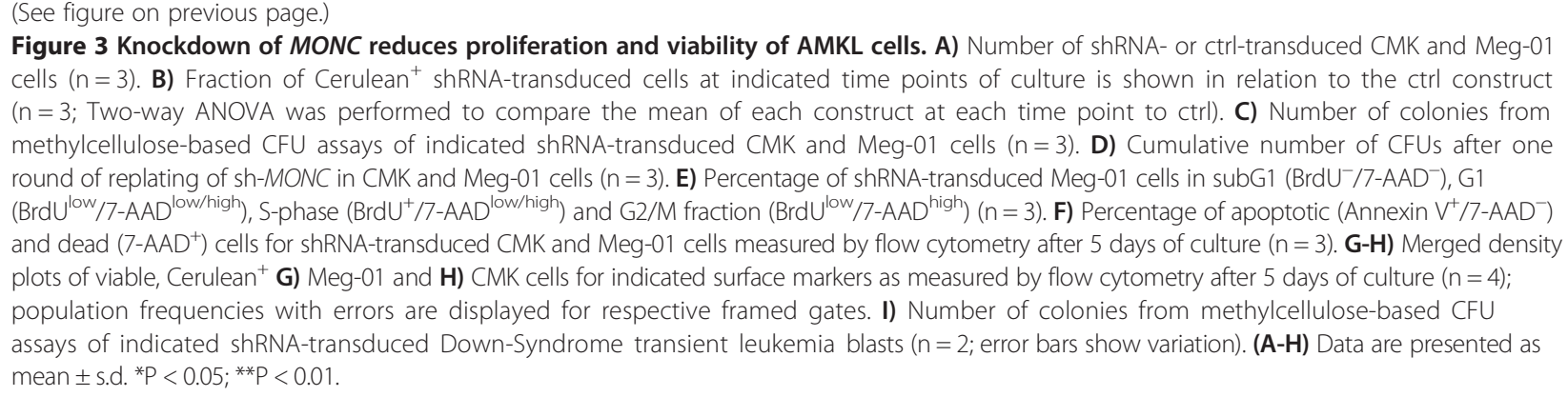

\section{Design and cloning of a lentiviral lincRNA expression vector}

To expand our knowledge about MONC in hematopoietic cells, we sought to ectopically express the lincRNA in CD34 ${ }^{+}$-HSPCs from healthy donors. Expression of the spliced lincRNA would also allow us to dissect its function from the intronic miRNAs. However, there are several challenges to consider. The transfection efficiency of plasmid DNA or RNA into CD34 ${ }^{+}$-HSPCs is very low [18]. Furthermore, transfected nucleic acids are diluted out during cell divisions. Thus, an integrating lentiviral vector stably overexpressing the transgene and a selection marker is advantageous. However, the lincRNA transcript from the lentiviral vector should be equivalent to the endogenous lincRNA. Transcription of adjacent proviral DNA due to improper termination downstream of the lincRNA transcript could alter the secondary structure of the lincRNA and therewith its function [19]. Thus, conventional vectors that are used for expression of protein coding genes are not suitable for studying the function of lncRNAs.

Therefore, we modified the widely used LeGO-CeB vector [20] by removing the murine U6 expression cassette for small RNAs and inserting a bovine growth hormone polyadenylation signal (BGH polyA) followed by the phosphoglycerate kinase (PGK) promoter. This created the LeGO-CeB/lnc vector, featuring a spleen focus-forming virus promoter (SFFV)-driven lincRNA expression cassette terminated by a polyA signal, and an independent PGK-driven marker cassette (Figure 4A). Although an insense oriented polyA signal interferes with viral genome RNA replication resulting in generally low titer yields, infective viral particles are generated in sufficient amounts to transduce primary cells as outlined below.

Spliced MIR100HG RNA has a length of $3082 \mathrm{nt}$ (NR_024430.1), precluding its cloning and evaluation with the described lentiviral vector. MONC has a length of 710 nt (ENST00000445461) (Additional file 3: Figure S3), which allowed successful cloning and production of functional lentiviral particles. Using genomic DNA (gDNA) of LeGO-CeB/lnc:MONC and LeGO-CeB/lnc:empty (vector) transduced HT1080 cells, we could confirm genomic integration of both vectors by PCR (Figure 4B, left gel charts). PCR using a forward primer (fwd2) binding to the MONC insert and a reverse primer binding to the downstream PGK promoter (rev1) validated the presence of $M O N C$ proviral DNA in the genome of MONC-transduced cells only (Figure 4B, left gel charts). RT-PCR with the same primer pair on cDNA of transduced HT1080 cells could not detect a corresponding transcript. In contrast, RT-PCR with a primer pair binding to MONC detected expression of the transgene, demonstrating that transcription of the lincRNA from the SFFV promoter was efficiently terminated by the polyA signal before the PGK promoter. qRT-PCR showed 40-fold upregulation of $M O N C$ expression in LeGO-CeB/lnc-MONCtransduced HT1080 cells (Figure 4B, right graph). Hence, we engineered a lentiviral lncRNA expression vector, LeGO$\mathrm{CeB} / \mathrm{lnc}$, which was validated to produce integrationcompetent virus and to express the lncRNA insert without vector-derived RNA.

\section{Ectopic MONC interferes with myeloid differentiation of HSPCs}

Next we overexpressed MONC in cord-blood (CB) CD34 ${ }^{+}$-HSPCs to determine its impact on hematopoietic lineage decisions. qRT-PCR in transduced HSPCs revealed more than 500-fold increased MONC levels (Figure 5A). This expression levels are comparable with the leukemic setting, as MONC levels are $~ 450$-fold elevated in CMK cells compared to CD34 ${ }^{+}$-HSPCs (Additional file 4: Figure S4). In CFU-megakaryocyte (CFU-MK) assays, the number of colonies was slightly reduced upon ectopic MONC expression (Figure 5B). Concordantly, in methocellulose-based myeloid CFU-assays MONC led to a decrease of granulocytic CFU-Gs, while erythroid BFU-Es were expanded (Figure 5C). However, in both CFU assays the total number of colonies was not significantly changed. Interestingly, culturing of HSPCs in a growth medium promoting multilineage progenitor expansion resulted in a more than 2-fold increase of $\mathrm{CD} 117^{+} / \mathrm{CD}^{+} 1^{+}$erythroid progenitor cells by $\mathrm{MONC}$ (Figure 5D). Strikingly, the percentage of $\mathrm{CD}_{13}{ }^{+}$ 


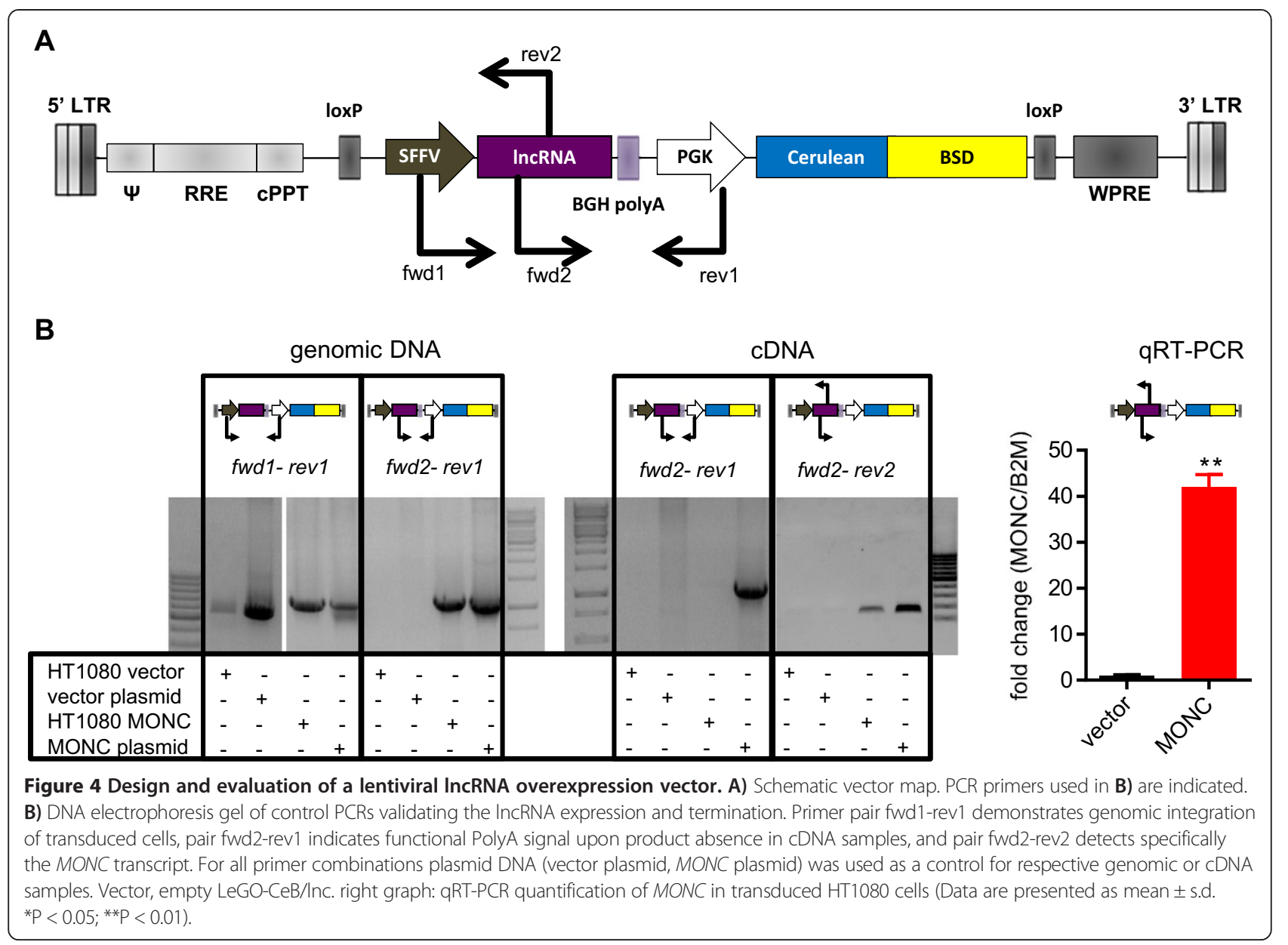

myelomonocytic progenitors was strongly reduced by MONC (Figure 5E). In liquid cultures promoting megakaryocytic and erythroid differentiation, we noted a switch in lineage decision. This was evident by a sharp increase of $\mathrm{CD} 6^{+} / \mathrm{CD} 235 \mathrm{a}^{+}$erythroid cells (Figure $5 \mathrm{~F}$ ) and decrease of $\mathrm{CD}_{4} 1^{+} / \mathrm{CD}_{4} 2 \mathrm{~b}^{+}$megakaryocytes (Figure $5 \mathrm{G}$ ). These data are in concordance with the BFU-E expansion and CFU-MK reduction in the CFU-assays.

In conclusion, enforced MONC expression in normal HSPCs changes the lineage bias towards the erythroid compartment and leads to the expansion of immature erythroid progenitor cells.

\section{MONC and MIR100HG are located in the nucleus}

To determine the subcellular localization, we applied RNA fluorescence in situ hybridization (RNA-FISH) to capture endogenous MONC and MIR100HG signals by locked nucleic acids (LNA) probes in CMK cells. Both MONC and MIR100HG probes showed predominantly a textured staining of nuclear areas (Figure 6A), as compared to polyadenylated mRNA (positive control). To confirm this localization pattern by an alternative method, we applied subcellular RNA fractionation followed by qRT-
PCR to calculate a cytoplasma:nucleus ratio. As expected beta-2 microglobulin (B2M) mRNA showed a clear cytoplasmic localization, while both MONC and MIR100HG transcripts showed a strong prevalence for nuclear localization (Figure 6B).

\section{Discussion}

Here we show the predominant expression of lincRNAs MIR100HG and MONC in HSPCs and erytroid/ megakaryocytic cells and their dysregulation in megakaryoblastic leukemia. The growth of AMKL cells was dependent on the continuous expression of both lincRNAs. Enforced expression of spliced MONC in normal HSPCs led to the predominant differentiation along the erythroid lineage and expansion of $\mathrm{CD} 117^{+} / \mathrm{CD} 71^{+}$immature erythroid progenitor cells at the expense of myeloid and megakaryocytic differentiation. Favoring fast growing progenitor stages by MONC might therefore provide a context for malignant transformation. Thus, it seems unlikely that those lincRNAs act merely as host genes or byproducts of miR-99a/100 $\sim 125 b$ cluster transcription by providing a Polymerase II promoter, as exemplified for the $m i R-31$ locus in breast cancer [21]. 


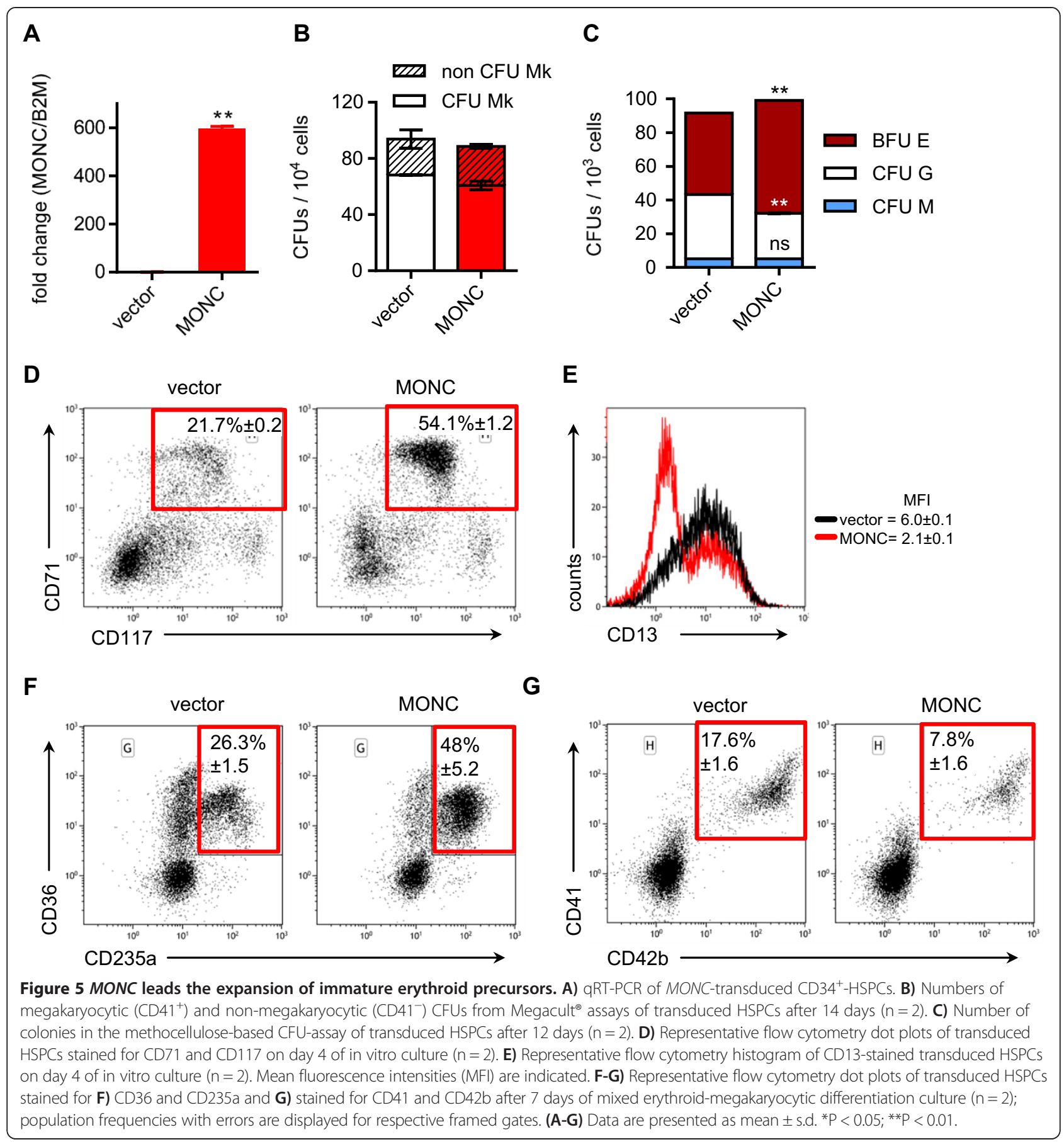

Results of RNA-FISH and qRT-PCR on fractionated RNA pointed towards a nuclear localization of MONC and MIR100HG. Recently an interesting hypothesis regarding the biological function of lncRNAs suggested that lncRNAs serve as subcellular address codes for other biomolecules [22]. Especially the nucleus with its higher order structures is an organelle suitable for lncRNA-directed spatial organization. This is particularly reflected by several lncRNAs interacting with chromatin remodelers to recruit them to specific genomic loci or subnuclear sites. E.g., Air mediates silencing of paternal alleles of multiple genes, Xist controls inactivation of one $\mathrm{X}$ chromosome in females, and Kcnq1ot1 regulates imprinting of placental genes. All three lncRNAs act by allele-specific directing of PRC2 or G9a, thereby leading to histone methylation of $\mathrm{H} 3 \mathrm{~K} 27 \mathrm{me} 3$ or $\mathrm{H} 3 \mathrm{~K} 9 \mathrm{me} 3$ [23-25]. Meanwhile, a compelling discovery in Drosophila unravelled the distinction of five principal chromatin 


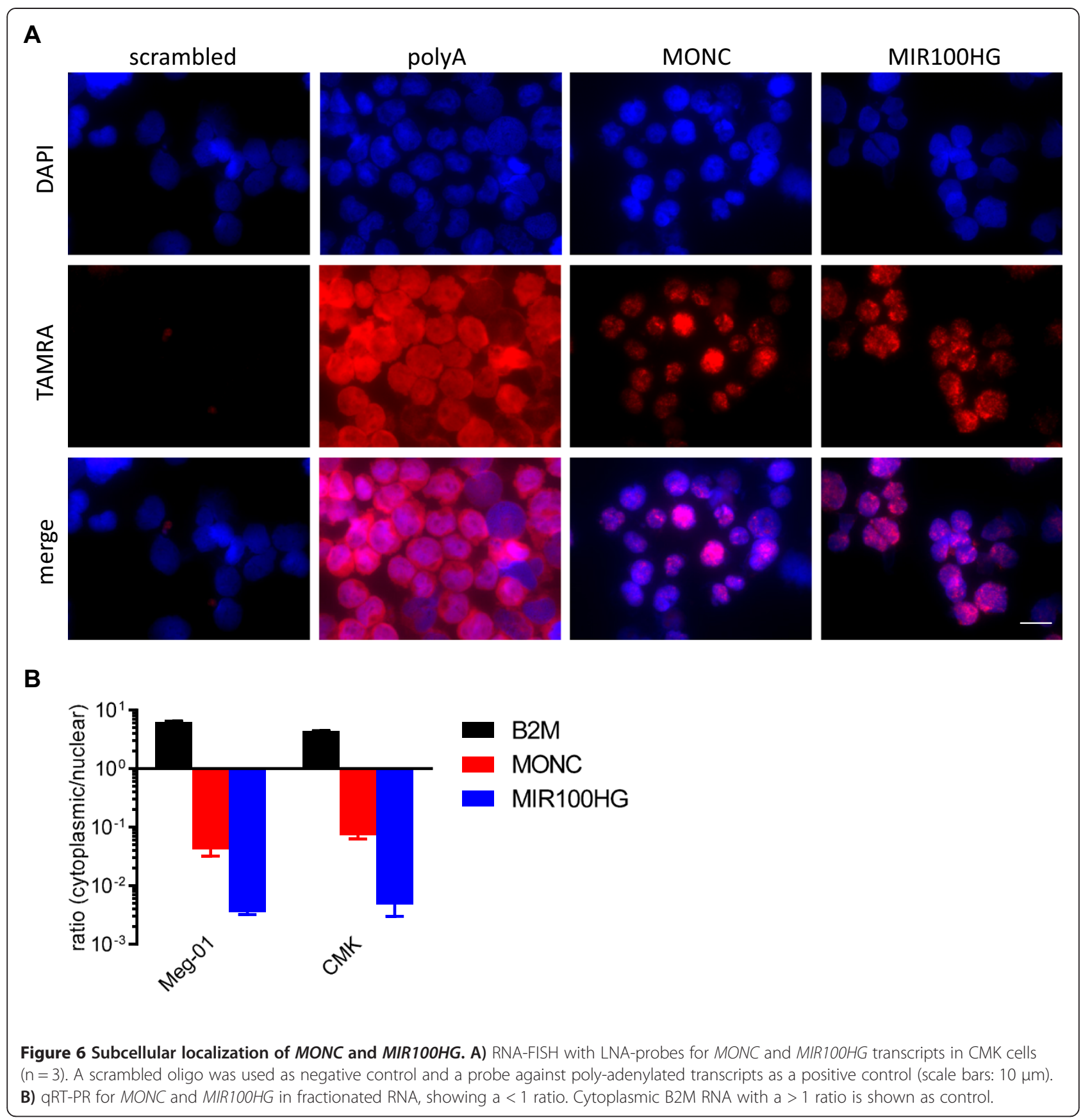

types by protein components, which form separate domains [26]. Recently this model became complemented by computational analysis of genome-wide epigenetic marks distribution to 4 principal chromatin types with virtually identical classification [27]. With this the genome can be compared with the design of a roadmap, where districts are defined by chromatin-bound proteins and epigenetic marks, lncRNAs form the street names and the gene loci regulatory sequences represent the house numbers. The fluorescence signals for both lincRNA probes show a broad, irregular dispersion rather than singular site distribution over the nucleus. This may indicate a contribution of either host gene to a ternary chromatin modifying or remodeling complex acting at multiple nuclear domains. The chromatin modifying SWI/SNF complex subunit BRG1 is associated with melanoma progression [28]. However, the lncRNA SChLAP1 imparts functioning of SWI/SNF complexes contributing to development of lethal prostate cancers [29]. Repression of the tumor suppressor INK4b-ARF-INK4a locus by ANRIL lncRNA is mediated by both Polycomb repressive complex-1 (PRC1) and PRC2, increasing the likelihood of oncogenesis [30]. 
Meanwhile a WDR5 mutant defective in RNA binding fails to activate gene expression in embryonic stem cells by the Trithorax Group/Mixed-Lineage-Leukemia complex [31]. Further research identifying protein interaction partners and pinpointing precise subnuclear areas and DNA target sequences of MONC and MIR100HG is warranted.

\section{Conclusions}

This study characterizes for the first time lincRNAs during megakaryopoiesis and AMKL. MONC and MIR100HG, the human $m i R-99 a / 100 \sim 125 b$ cluster host genes, reside in the nuclear cell compartment, where they play a role in the regulation of erythro-megakaryocytic development. In AMKL they contribute to the maintenance of leukemic growth. Given the central role of miR-99 125 polycistron miRNAs in AML, advanced understanding of the gene products from these loci will ultimately lead to therapy improvements of this aggressive malignancy.

\section{Methods}

\section{Patient samples and cell lines}

The AML-'Berlin-Frankfurt-Münster' Study Group (AMLBFM-SG, Hannover, Germany) provided all patient samples. CB HSPCs from donors were positively selected by labeling CD34 expressing cells with magnetic cell-sorting beads (Miltenyi Biotech). Culture conditions for maintenance, megakaryocytic or megakaryocytic/erythroid in vitro differentiation of CD34 ${ }^{+}$-HSPCs were described elsewhere [32-34]. Cell lines (CMK, Meg-01, K562, HT1080 and 293T) were purchased from the German National Resource Center for Biological Material (DSMZ) and maintained under recommended conditions. All investigations had been approved by the local Ethics Committee.

\section{Constructs and lentivirus}

Cloning of shRNAs into a modified LeGO vector was performed as previously described [32,35]. A non-silencing shRNA in the miR-30 backbone (Open Biosystems) was subcloned to the LeGO vector and used as control (referred to as non-silencing miRNA). ShRNAs against human MONC were obtained from Open Biosystems (Clone IDs V2LHS_206411, V2LHS_208623) or designed by TRC (http://www.broadinstitute.org/rnai/public/) and subcloned into the LeGO miR-30 backbone construct. Stable lincRNA overexpression was achieved using a novel modified LeGO vector, LeGO-CeB/lnc. Briefly, we removed the murine U6 promoter by XhoI and XbaI digestion with subsequent end filling by a proof reading polymerase (Phusion II, Finnzymes) and religation. Next we inserted the PGK promoter from pMSCV-Puro-IRESGFP [36] retrovector with a 5' 20 nt spacer containing NsiI site into the BamHI site adjacent to the SFFV promoter. A BGH polyA signal from pMIRREPORT was inserted into NsiI. An oligo with the MCS for lncRNA fragments was inserted via NotI between SFFV and polyA. The MONC isoform MIR99AHG-iso6 (ENST00000445461) was synthesized by GeneArt (lifetech). Lentiviral supernatant was generated and collected using standard protocols as described [32].

\section{Transduction and hematopoietic assays}

CD34+ HSPCs were lentivirally transduced on RetroNectincoated (Takara) plates as described [32]. Methylcellulosebased (Methylcellulose Base and Complete, RnD Systems) and collagen-based (Megacult ${ }^{\oplus}$, Stem Cell Technologies) colony-forming assays were carried out according to the manufacturers' instructions. Serial replating was performed as described previously [33]. Cumulative colony numbers were calculated with the following equation: $\mathrm{CFU}(\mathrm{k})=\sum_{\mathrm{n}=1}^{\mathrm{k}} \mathrm{CFU}_{\mathrm{n}}$, where $\mathrm{CFU}_{\mathrm{n}}=$ number of counted colonies from respective platings (n). Note that if a fraction of cells $(\mathrm{f})$ from the $1^{\text {st }}$ plating was replated for the $2^{\text {nd }}$ plating, then $\mathrm{CFU}(\mathrm{k})=\mathrm{CFU}_{1}+\frac{\mathrm{CFU}_{2}}{\mathrm{f}_{1}}$.

\section{Cell growth, cell cycle and apoptosis assays}

Apoptosis was detected with the Annexin V Apoptosis Detection Kit II (Becton Dickinson) and cell cycle was analyzed with the the BrDU Flow Kit (Becton Dickinson). All assays were performed according to the manufacturer's instructions. Growth competition assays were performed by mixing each transduced Cerulean + population 1:1 with a control population expressing eGFP.

\section{Flow Cytometry and Cell Sorting}

Transduced HSPCs were sorted based on GFP-expression. Flow Cytometry was performed on a Navios 10/3 (Beckman Coulter). Kaluza 1.2 (Beckman Coulter) was used for data analysis. Staining and measuring were performed according to standard protocols and as described previously using the antibodies PE-CD42b, PC5.5-CD13, PC7-CD41, PC7-CD117, AlexaFluor 750-CD235a (all Beckman Coulter), APC-CD36, APC-CD42b (both Becton Dickinson) and PacificBlue-CD71 (Exbio) [14].

\section{RNA isolation and Quantitative real-time PCR (qRT-PCR)}

Standard RNA isolation, cDNA synthesis and mRNA qRT-PCR were done as described [14]. qRT-PCR primer sequences are available upon request, B2M was used as reference gene. MiRNA-Detection was performed with TaqMan miRNA assays (ABI), RNU44 was used as reference gene. All data were analyzed in a StepOnePlus Cycler (ABI) using the geNORM $\Delta \Delta \mathrm{Ct}$ equations. RNA fractionation into cytoplasmic and nuclear lysates was done by PARIS Kit (Ambion, lifetech) according to manufacturers' instructions. 


\section{5'-RACE PCR}

For rapid amplification of cDNA ends the GeneRacer ${ }^{\oplus}$ Kit with SuperScript ${ }^{\ominus}$ III RT and Zero Blunt ${ }^{\ominus}$ TOPO $^{\circ}$ PCR Cloning Kit for Sequencing (Invitrogen) were used. The 5' ends were amplified by nested PCR using HotStar Mastermix (Qiagen) and Phusion Polymerase (Finnzymes). Primers and sequenced clones are available upon request.

\section{RNA Fluorescence in situ hybridization}

RNA detection was performed according to de PlanellSaguer [37]. Specifically, the LNA ISH with following tyramide signal amplification protocol was used. CMK cells were prepared as cytospins from fresh mock cultures. All TAMRA-conjugated LNA probes were designed and synthesized by Exiqon. Fluorescence microscopy was carried out on a BZ9000 (Keyence), data analysis was performed with Biorevo Software (Keyence).

\section{Statistical analysis}

Statistical evaluation between two groups was carried out using Student's t-test and for more than two groups by 2-way ANOVA with Tukey's or Sidak's post-hoc analysis. The level of significance was set at $\mathrm{P}<0.05$. All data are presented as mean \pm s.d. Calculations were performed using GraphPad Prism 6.

\section{Additional files}

Additional file 1: Figure S1. A) qRT-PCR of MIR100HG in shRNA-transdued CMK cells. B) Number of shRNA- or ctrl-transduced K562 cells. C) Growth competition assay. The fraction of Cerulean ${ }^{+}$shRNA-transduced cells at indicated time points of culture is shown in relation to the ctrl construct.

Additional file 2: Figure S2. A) $q R T-P C R$ of MONC in shRNA-transdued CMK cells. B) Number of shRNA- or ctrl-transduced K562 cells. C) Well pictures of automated microscopy assays in indicated cell lines on day 4 (scale bar: $200 \mu \mathrm{m})(\mathrm{n}=1)$. D) Number of colonies from methylcellulose-based colony-forming assays of sh-MONC transduced K562, CMK and M-07 cells.

Additional file 3: Figure S3. Sequence of MONC iso-6 transcript (ENST00000445461.2) cloned into LeGO-CeB/Inc vector.

Additional file 4: Figure S4. Basal expression levels of MIR100HG and MONC in CD34 ${ }^{+}$HSPCs compared to CMK cells as determined by qPCR.

\section{Competing interests}

The authors declare that they have no competing interests.

\section{Authors' contributions}

SE, AS, and FS performed molecular studies, statistical analyses and data interpretation. VRT performed experiments. SE and JHK analyzed and interpreted results, supervised the study and wrote the manuscript. JHK designed the research. DR provided lab space and patient material. All authors read and approved the final manuscript.

\section{Acknowledgements}

We thank Prof. Axel Schambach for critical inputs on vector design and Dr Rudolf Bauerfeind for help with microscopy and image processing. This work was supported by a grant to J.H.K. from the German Research Foundation (KL-2374/2-1) and the German Cancer Aid (DKH, 109251). J.H.K is a fellow of the Emmy Noether-Programme from the DFG (KL-2374/2-1). A.S. was supported by the DKH (110108).
Received: 7 April 2014 Accepted: 3 July 2014

Published: 15 July 2014

\section{References}

1. Dinger ME, Amaral PP, Mercer TR, Mattick JS: Pervasive transcription of the eukaryotic genome: functional indices and conceptual implications. Brief Funct Genomic Proteomic 2009, 8:407-423.

2. Katayama S, Tomaru Y, Kasukawa T, Waki K, Nakanishi M, Nakamura M, Nishida H, Yap CC, Suzuki M, Kawai J, Suzuki H, Carninci P, Hayashizaki Y, Wells C, Frith M, Ravasi T, Pang KC, Hallinan J, Mattick J, Hume DA, Lipovich L, Batalov S, Engstrom PG, Mizuno Y, Faghihi MA, Sandelin A, Chalk AM, Mottagui-Tabar S, Liang Z, Lenhard B, et al: Antisense transcription in the mammalian transcriptome. Science 2005, 309:1564-1566.

3. Nakaya HI, Amaral PP, Louro R, Lopes A, Fachel AA, Moreira YB, El Jundi TA, da Silva AM, Reis EM, Verjovski-Almeida S: Genome mapping and expression analyses of human intronic noncoding RNAs reveal tissue-specific patterns and enrichment in genes related to regulation of transcription. Genome Biol 2007, 8:R43.

4. Penny GD, Kay GF, Sheardown SA, Rastan S, Brockdorff N: Requirement for Xist in X chromosome inactivation. Nature 1996, 379:131-137.

5. Yildirim E, Kirby JE, Brown DE, Mercier FE, Sadreyev RI, Scadden DT, Lee JT: Xist RNA is a potent suppressor of hematologic cancer in mice. Cell 2013, 152:727-742.

6. Rinn JL, Kertesz M, Wang JK, Squazzo SL, Xu X, Brugmann SA, Goodnough LH, Helms JA, Farnham PJ, Segal E, Chang HY: Functional demarcation of active and silent chromatin domains in human HOX loci by noncoding RNAs. Cell 2007, 129:1311-1323.

7. Tsai MC, Manor O, Wan Y, Mosammaparast N, Wang JK, Lan F, Shi Y, Segal E, Chang HY: Long noncoding RNA as modular scaffold of histone modification complexes. Science 2010, 329:689-693.

8. Wang KC, Yang YW, Liu B, Sanyal A, Corces-Zimmerman R, Chen Y, Lajoie BR, Protacio A, Flynn RA, Gupta RA, Wysocka J, Lei M, Dekker J, Helms JA, Chang HY: A long noncoding RNA maintains active chromatin to coordinate homeotic gene expression. Nature 2011, 472:120-124.

9. Feldstein O, Nizri T, Doniger T, Jacob J, Rechavi G, Ginsberg D: The long non-coding RNA ERIC is regulated by E2F and modulates the cellular response to DNA damage. Mol Cancer 2013, 12:131.

10. Estey E, Dohner H: Acute myeloid leukaemia. Lancet 2006, 368:1894-1907.

11. Hasle $\mathrm{H}$, Clemmensen $\mathrm{IH}$, Mikkelsen M: Risks of leukaemia and solid tumours in individuals with Down's syndrome. Lancet 2000, 355:165-169.

12. Athale UH, Razzouk BI, Raimondi SC, Tong X, Behm FG, Head DR, Srivastava DK, Rubnitz JE, Bowman L, Pui CH, Ribeiro RC: Biology and outcome of childhood acute megakaryoblastic leukemia: a single institution's experience. Blood 2001, 97:3727-3732.

13. Creutzig U, Reinhardt D, Diekamp S, Dworzak M, Stary J, Zimmermann M: AML patients with Down syndrome have a high cure rate with AML-BFM therapy with reduced dose intensity. Leukemia 2005, 19:1355-1360.

14. Klusmann JH, Li Z, Bohmer K, Maroz A, Koch ML, Emmrich S, Godinho FJ, Orkin SH, Reinhardt D: miR-125b-2 is a potential oncomiR on human chromosome 21 in megakaryoblastic leukemia. Genes Dev 2010, 24:478-490.

15. Emmrich S, Rasche M, Schoning J, Reimer C, Keihani S, Maroz A, Xie Y, Li Z, Schambach A, Reinhardt D, Klusmann JH: miR-99a/100 125b tricistrons regulate hematopoietic stem and progenitor cell homeostasis by shifting the balance between TGFbeta and Wnt signaling. Genes Dev 2014, 28:858-874.

16. Chen PS, Su JL, Cha ST, Tarn WY, Wang MY, Hsu HC, Lin MT, Chu CY, Hua KT, Chen CN, Kuo TC, Chang KJ, Hsiao M, Chang YW, Chen JS, Yang PC, Kuo ML: miR-107 promotes tumor progression by targeting the let-7 microRNA in mice and humans. J Clin Invest 2011, 121:3442-3455.

17. Hagan JP, Piskounova E, Gregory RI: Lin28 recruits the TUTase Zcchc11 to inhibit let-7 maturation in mouse embryonic stem cells. Nat Struct Mol Biol 2009, 16:1021-1025.

18. Maurisse R, De Semir D, Emamekhoo H, Bedayat B, Abdolmohammadi A, Parsi H, Gruenert DC: Comparative transfection of DNA into primary and transformed mammalian cells from different lineages. BMC Biotechnol 2010, 10:9.

19. Wan G, Liu Y, Han C, Zhang X, Lu X: Noncoding RNAs in DNA repair and genome integrity. Antioxid Redox Signal 2014, 20:655-677.

20. Weber K, Bartsch U, Stocking C, Fehse B: A multicolor panel of novel lentiviral "gene ontology" (LeGO) vectors for functional gene analysis. Mol Ther 2008, 16:698-706. 
21. Augoff $K$, McCue B, Plow EF, Sossey-Alaoui K: miR-31 and its host gene InCRNA LOC554202 are regulated by promoter hypermethylation in triple-negative breast cancer. Mol Cancer 2012, 11:5.

22. Batista PJ, Chang HY: Long noncoding RNAs: cellular address codes in development and disease. Cell 2013, 152:1298-1307.

23. Kay GF, Penny GD, Patel D, Ashworth A, Brockdorff N, Rastan S: Expression of Xist during mouse development suggests a role in the initiation of $X$ chromosome inactivation. Cell 1993, 72:171-182.

24. Latos PA, Pauler FM, Koerner MV, Senergin HB, Hudson QJ, Stocsits RR, Allhoff W, Stricker SH, Klement RM, Warczok KE, Aumayr K, Pasierbek P, Barlow DP: Airn transcriptional overlap, but not its IncRNA products, induces imprinted Igf2r silencing. Science 2012, 338:1469-1472.

25. Mancini-Dinardo D, Steele SJ, Levorse JM, Ingram RS, Tilghman SM: Elongation of the Kcnq1ot1 transcript is required for genomic imprinting of neighboring genes. Genes Dev 2006, 20:1268-1282.

26. Filion GJ, van Bemmel JG, Braunschweig U, Talhout W, Kind J, Ward LD, Brugman W, de Castro IJ, Kerkhoven RM, Bussemaker HJ, van Steensel B: Systematic protein location mapping reveals five principal chromatin types in Drosophila cells. Cell 2010, 143:212-224.

27. Julienne H, Zoufir A, Audit B, Arneodo A: Human genome replication proceeds through four chromatin states. PLoS Comput Biol 2013, 9:e1003233.

28. Saladi SV, Keenen B, Marathe HG, Qi H, Chin KV, de la Serna I: Modulation of extracellular matrix/adhesion molecule expression by BRG1 is associated with increased melanoma invasiveness. Mol Cancer 2010, 9:280.

29. Prensner JR, lyer MK, Sahu A, Asangani IA, Cao Q, Patel L, Vergara IA, Davicioni E, Erho N, Ghadessi M, Jenkins RB, Triche TJ, Malik R, Bedenis R, McGregor N, Ma T, Chen W, Han S, Jing X, Cao X, Wang X, Chandler B, Yan W, Siddiqui J, Kunju LP, Dhanasekaran SM, Pienta KJ, Feng FY, Chinnaiyan AM: The long noncoding RNA SChLAP1 promotes aggressive prostate cancer and antagonizes the SWI/SNF complex. Nat Genet 2013, 45:1392-1398.

30. Aguilo F, Zhou MM, Walsh MJ: Long noncoding RNA, polycomb, and the ghosts haunting INK4b-ARF-INK4a expression. Cancer Res 2011 71:5365-5369.

31. Yang YW, Flynn RA, Chen Y, Qu K, Wan B, Wang KC, Lei M, Chang HY: Essential role of IncRNA binding for WDR5 maintenance of active chromatin and embryonic stem cell pluripotency. Elife 2014, 3:e02046.

32. Emmrich S, Henke K, Hegermann J, Ochs M, Reinhardt D, Klusmann JH: miRNAs can increase the efficiency of ex vivo platelet generation. Ann Hematol 2012, 91:1673-1684.

33. Klusmann JH, Godinho FJ, Heitmann K, Maroz A, Koch ML, Reinhardt D, Orkin SH, Li Z: Developmental stage-specific interplay of GATA1 and IGF signaling in fetal megakaryopoiesis and leukemogenesis. Genes Dev 2010, 24:1659-1672.

34. Stankov MV, El Khatib M, Kumar TB, Heitmann K, Panayotova-Dimitrova D, Schoening J, Bourquin JP, Schweitzer N, Leverkus M, Welte K, Reinhardt D, Li Z, Orkin SH, Behrens GM, Klusmann JH: Histone deacetylase inhibitors induce apoptosis in myeloid leukemia by suppressing autophagy. Leukemia 2014, 28:577-588

35. Weber K, Mock U, Petrowitz B, Bartsch U, Fehse B: Lentiviral gene ontology (LeGO) vectors equipped with novel drug-selectable fluorescent proteins: new building blocks for cell marking and multi-gene analysis. Gene Ther 2010, 17:511-520.

36. Dickins RA, Hemann MT, Zilfou JT, Simpson DR, Ibarra I, Hannon GJ, Lowe SW: Probing tumor phenotypes using stable and regulated synthetic microRNA precursors. Nat Genet 2005, 37:1289-1295.

37. Planell-Saguer M, Rodicio MC, Mourelatos Z: Rapid in situ codetection of noncoding RNAs and proteins in cells and formalin-fixed paraffin-embedded tissue sections without protease treatment. Nat Protoc 2010, 5:1061-1073.

\section{doi:10.1186/1476-4598-13-171}

Cite this article as: Emmrich et al:: LincRNAs MONC and MIR100HG act as oncogenes in acute megakaryoblastic leukemia. Molecular Cancer 2014 13:171.

\section{Submit your next manuscript to BioMed Central and take full advantage of:}

- Convenient online submission

- Thorough peer review

- No space constraints or color figure charges

- Immediate publication on acceptance

- Inclusion in PubMed, CAS, Scopus and Google Scholar

- Research which is freely available for redistribution 